\title{
VARIABILIDAD ESPACIO TEMPORAL DE LA DERIVA DE LOS VIENTOS OESTES MEDIANTE VIENTOS SATELITALES (QUIKSCAT)
}

\section{SPATIAL AND TEMPORAL VARIABILITY OF WESTERLY WIND DRIFT BY SATELLITE WINDS (QUIKSCAT)}

\author{
Tamara Pardo Márquez ${ }^{1}$ y Luis Soto Mardones ${ }^{2}$ \\ ${ }^{1}$ Facultad de Ciencias, Departamento de Matemáticas, Universidad de Bío-Bío, Av. Collao 1202, \\ casilla 5-C, CP 4081112 Concepción, Chile. \\ ${ }^{2}$ Facultad de Ciencias, Departamento de Física, Universidad de Bío-Bío, Av. Collao 1202, \\ casilla 5-C, CP 4081112 Concepción, Chile.
}

\begin{abstract}
RESUMEN
Mediante el uso de 110 imágenes mensuales (1999-2008) del esfuerzo del viento proveniente del satélite Quikscat se analizó la zona donde divergen los vientos hacia el ecuador y hacia los polos. En las cercanías del borde oriental del Pacífico, tanto para el norte como para el sur, se estudió el desplazamiento de las zonas de divergencia de los vientos (DZD) mediante una serie de tiempo versus posición. Mediante análisis estadístico se encontró que el DZD está influenciado por señales de alta frecuencia (periodo de 3 meses), estacional (periodo de 12 meses) e interanual (periodos superior a dos años) asociado al evento cálido de El Niño. La señal más robusta de la serie es la estacional, mostrando que el DZD de la región norte está fuertemente acoplado a la región sur. Además, el DZD de la región norte muestra un mayor desplazamiento estacional, asociado posiblemente a la forma de la línea de costa ya que la región sur está sometida a forzantes de origen polar.
\end{abstract}

PALABRA ClAVEs: estacional, desplazamiento, divergencia, interanual, vientos.

\section{ABSTRACT}

By mean of 110 monthly images (1999-2008) of wind stress from Quikscat satellite, the divergence zone of equatorward and poleward winds was analyzed. At the surrounding area of the Pacific Eastern boundary, for both north and south, the displacement of the wind divergence zone (DDZ) was studied by a time serie versus position. The statistical analysis reveals that DDZ is influenced by high frequency (3 months), seasonal (12 months) and interannual signals (> 2 years), the last associated to warm event of El Niño. The most predominant signal was the seasonal, showing DDZ in the northern zone is highly coupled with the DDZ in the south. Furthermore, the DDZ of the northern zone showed a major seasonal displacement in comparison to the southern area, likely associated to the shape of coastline, since the southern zone is influenced by polar signals.

Keywords: Seasonal, Displacement, Divergence, Interannual, Winds.

\section{INTRODUCCION}

Entre los $30^{\circ}$ y $60^{\circ}$ surgen los vientos del Oeste, situado entre los vientos polares del este y los predominantes del oeste, donde se forma el flanco polar de los anticiclones subtropicales, debido al gradiente de presión entre los anticlones y las depresiones situadas a mayor latitud (Rutllant, 2004). En el hemisferio norte son muy variables ya que en estas latitudes el movimiento del aire se ve afectado por núcleos de alta y baja presión, vinculado a las ondas de Rossby que viajan del Oeste a Este. Por otro lado la irregularidad que, en el hemisferio norte, suponen los continentes enmascara 
el flujo zonal del Oeste. Los vientos oeste del hemisferio sur son mas fuertes y de dirección mas constante debido a la menor extensión de los océanos (Rutllant, 2004).

En el borde oriental del Pacifico sur interaccionan el Anticiclón Atmosférico Subtropical Suroriental, La Baja Polar y la Baja Costera (Saavedra y Foppiano, 1992). Estos centros de presión predominan sobre la circulación general de vientos en el Pacífico Suroriental. Y desde el punto de vista oceanográfico, este tren de vientos genera en el borde occidental la Corriente de Deriva de los Vientos Oeste la cual se traslada hacia el borde oriental (Reid, 1965; Wyrtki, 1975; Silva y Neshyba, 1979).

El trabajo tiene la necesidad de estudiar un parámetro atmosférico que detona la dinámica superficial del océano, además, de ser un tema poco estudiado dado su alta importancia en los procesos de afloramiento y hundimiento del océano en los bordes orientales y de ser el principal responsables de las corriente de deriva de los oestes.

\section{MATERIALES Y METODOS}

Datos Esfuerzo del viento 1999-2008, Satélite QuikSCAT (Propulsion Laboratory, htp.) de resolución espacial $0.5^{\circ}$ y temporal de cada 12 horas. A la secuencia de imágenes del esfuerzo del viento se aplicaron los siguientes análisis estadísticos: Funciones Empíricas Ortogonales, Función de Autocorrelación, Correlación Cruzada y Función Transformada de Wavelet Continua [Daubechies, 1992, Kaiser, 1994, Burrus et.al., 1998]. Para el estudio de las derivas de los vientos oestes se separaron en dos áreas: Hemisferio Norte $\left(25^{\circ} \mathrm{N}\right.$ $60^{\circ} \mathrm{N}$ y $\left.140^{\circ} \mathrm{W}-120^{\circ} \mathrm{W}\right)$ y Hemisferio Sur $\left(25^{\circ} \mathrm{S}-\right.$ $60^{\circ} \mathrm{N}$ y $\left.80^{\circ} \mathrm{W}-70^{\circ} \mathrm{W}\right)$. El periodo de estudio comienza en Agosto de 1999 hasta octubre de 2008 (110 imágenes del esfuerzo del viento mensual).

\section{RESULTADOS}

Se realiza la climatología en ambos hemisferios, donde el área está dominada por el cinturón de los vientos oeste (oestes) en la cual se alternan altas y bajas presiones móviles, que perturban localmente el frente polar (Rutllant, 2004). En ambas zonas, se produce una zona de divergencia del esfuerzo del viento, en el cual es posible definir claramente la posición (en función de la latitud) donde divergen los esfuerzo del viento, es decir, donde se separan los vientos en una rama hacia las regiones polares y la otra rama hacia bajas latitudes. Con el objetivo de facilitar la comparación de ambos hemisferios se invirtió la región sur quedando ambas regiones a la misma latitud (Fig. 1). Además, se comparan los mapas en forma estacional, es decir, invierno del norte versus invierno austral y así consecutivamente. En la figura 1, muestra a modo de resumen solamente un mes asociado a cada estación, bajo este orden, se muestra para el periodo de invierno tanto para la región norte (diciembre) como para la región sur (junio).

Para los meses de invierno de ambos hemisferios, se observa claramente una rama del esfuerzo del viento que se desvía hacia las regiones polares y la otra rama hacia bajas latitudes. Además, el esfuerzo del viento presenta las mayores magnitudes hacia las zonas polares, aunque, las de mayor magnitud son en la región sur. En el caso del sector norte la zona de divergencia se desplazo entre $39^{\circ} \mathrm{N}$ (diciembre) < DZD $<43^{\circ} \mathrm{N}$ (febrero), mientras la región sur fluctúo $36^{\circ} \mathrm{S}$ (junio) $<\mathrm{DZD}$ $<38^{\circ} \mathrm{S}$ (agosto).

Para el periodo de primavera cuando los vientos se empiezan a intensificar en ambos hemisferios y cual ha sido reportado por diversos autores (Hickey, 1979; Beardsley et al., 1987; Bakun y Nelson, 1991; Schwerdtfeger 1976; Montecinos, 1991; Fuenzalida, 1992; Carvajal, 1994; Pizarro et al., 1994). La figura 1 muestra para los meses de marzo y septiembre asociado a ambos hemisferios que las mayores magnitudes se encuentran en la región sur. Y el desplazamiento de la zona de divergencia se desplazo entre $43^{\circ} \mathrm{N}$ (marzo) $<\mathrm{DZD}<50^{\circ} \mathrm{N}$ (mayo), mientras, la región sur fluctúo $40^{\circ} \mathrm{S}$ (septiembre) $<$ $\mathrm{DZD}<43^{\circ} \mathrm{S}$ (agosto).

En verano el esfuerzo del viento está representado en la figura 1 por los meses de junio (norte) y diciembre (sur), donde el DZD en la región norte alcanza su máximo desplazamiento hacia altas latitudes y la menor en la región sur. Y el desplazamiento de la zona de divergencia se desplazo entre $51^{\circ} \mathrm{N}$ (junio) $>\mathrm{DZD}>50^{\circ} \mathrm{N}$ (agosto), mientras, la región sur fluctúo $42^{\circ} \mathrm{S}$ (diciembre) $<$ $\mathrm{DZD}<46^{\circ} \mathrm{S}$ (febrero).

El periodo de otoño el DZD de ambas regiones se desplazan hacia las regiones y los desplazamientos de la zona de divergencia ocurre entre $41^{\circ} \mathrm{N}$ (noviembre) $>$ DZD $>40^{\circ} \mathrm{N}$ (mayo), mientras, la región sur fluctúo 
Variabilidad espacio temporal mediante vientos satelitales: PARdo, T. \& L. Soto.

$44^{\circ} \mathrm{S}$ (marzo) $>$ DZD $>41^{\circ} \mathrm{S}$ (mayo).

La climatología revela claramente el comportamiento estacional del DZD y concluyendo que la región norte muestra un mayor desplazamiento $\left(12^{\circ}\right)$ que la región sur $\left(10^{\circ}\right)$. Por otro lado, las máximas intensidades del esfuerzo de viento se producen en el periodo de primavera y verano y los mínimos en el periodo de invierno.

Con el objeto de examinar la variabilidad espacio temporal de los mapas del esfuerzo del viento en ambas regiones se aplicaron las funciones empíricas ortogonales (FEOs) y cuya aplicación sólo depende de consideraciones estadísticas (Barnett y Patzert, 1980; Kelly, 1985). Las FEOs permiten cuantificar la varianza total en modos ortogonales o independientes entre sí. De esta manera se obtienen los modos que contienen el mayor porcentaje de la varianza, que representan a las señales dominantes en la región de estudio, quedando en los modos inferiores las contribuciones asociadas con otro tipo de procesos o escalas. En este capitulo se discute solamente la componente vertical del esfuerzo del viento (meridional), debido a que la climatología reporta que la variabilidad del DZD varia latitudinalmente.
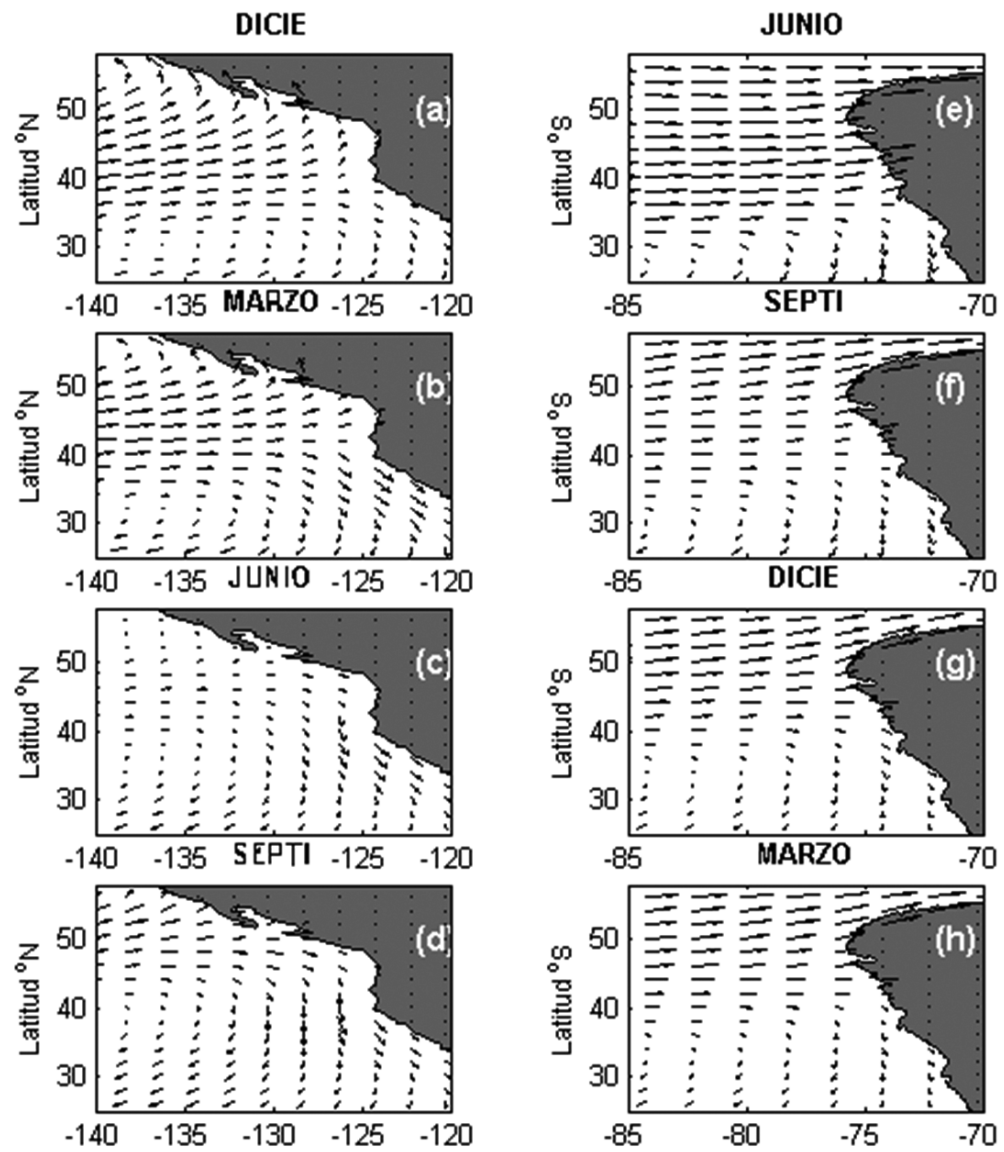

Figura 1. Climatología del esfuerzo del viento, sector norte (a, b, c, d) y sector sur (e, f, g, h).

FIGURE 1. Wind stress climatology, northern area (a, b, c, d), and southern area (e, f, g, h). 
Las figura 2.a y b, muestran para la región norte el primer modo del esfuerzo meridional y asociado al 69\% de la varianza explicada. La estructura espacial (Fig. 2.a) discrimina claramente la posición del DZD $\left(\sim 45^{\circ} \mathrm{N}\right)$, donde una rama apunta hacia zonas polares y la otra rama hacia bajas latitudes. Por otro lado, la estructura temporal (Fig. 2.b) muestra un comportamiento aproximadamente estacional, destacándose mayores amplitudes en 2001 y 2007.
De igual forma, el segundo modo asociado a un $15.83 \%$ de la varianza total, muestra que la estructura espacial (Fig. 2.c) nos indica la zona de mayor variabilidad del esfuerzo del viento meridional cuyo núcleo está centrado alrededor de los $45^{\circ} \mathrm{N}$, además, resalta la estacionalidad de la evolución temporal (Fig. 2.d). En resumen, la variabilidad espacio-temporal del DZD de la región norte es explicable a partir de los dos primeros modos de los FEOs.

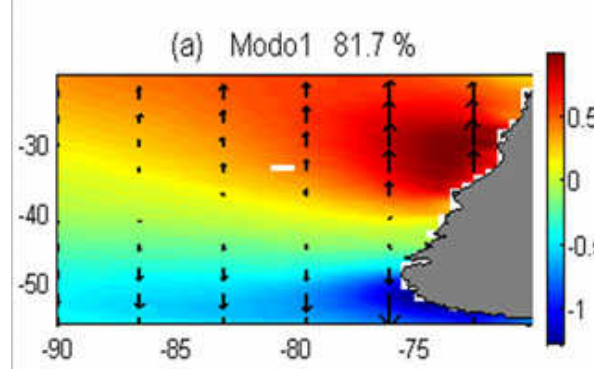

(c) Modo2 $7.7 \%$

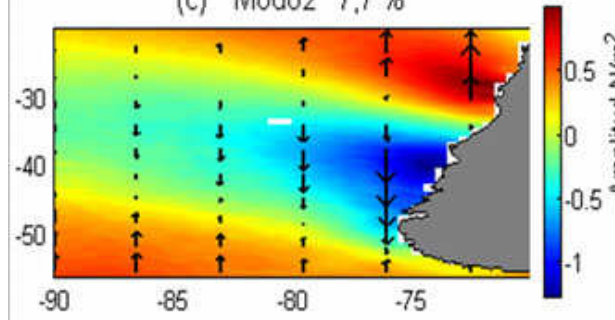

(b) Variabilidad Temporal del Esfuerzo del Viento Meridional del Modo1

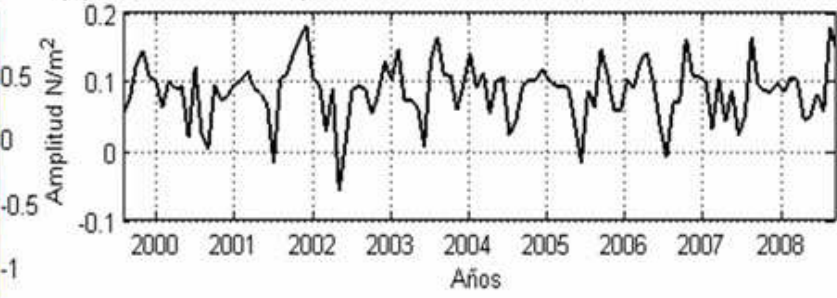

(d) Variabilidad Temporal del Esfuerzo del Viento Meridional del Modo2

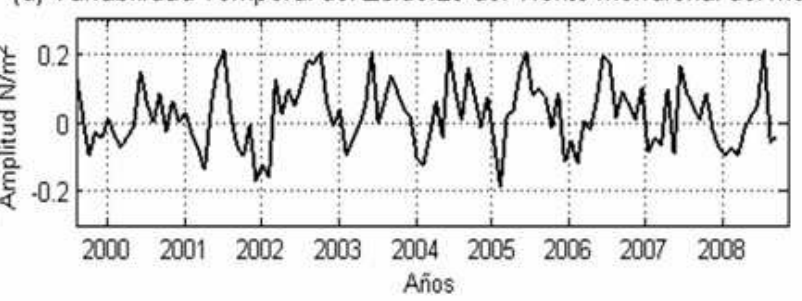

FIGURA 2. Funciones empíricas ortogonales para el esfuerzo del viento meridional, zona norte. Estructura espacial (a y c) y temporal (b y d) de los dos primeros modos.

FIGURE 2. Empirical orthogonal functions of meridional wind stress, northern area. Temporal (b and d) and spatial structure (a and c) of the two first modes.

Al aplicar las FEOs al esfuerzo del viento meridional de la región sur, las figuras 3 a y b muestran el primer modo que explica el $81.7 \%$ de la varianza total. La estructura espacial (Fig. 3.a) del primer modo rescata claramente la posición del cinturón de los vientos oestes $\left(\sim 47^{\circ} \mathrm{S}\right)$ y cuyo comportamiento temporal (Fig. 3.ab) evoluciona en forma aproximada mente estacional, aunque, destacan amplitudes de mayor magnitud (2002) asociado posiblemente a señales de otras escalas. Por otro lado, la estructura espacial (Fig. 3.c) del segundo modo rescata un núcleo costero que oscila entre los $35^{\circ} \mathrm{S}$ y $51^{\circ} \mathrm{S}$ denotando el desplazamiento estacional del DZD.

Con el fin de precisar como se desplaza en la región más costera el DZD, se extrajo a cada mapa de esfuerzo del viento la posición en función de la latitud, generando un conjunto de datos (tiempo versus latitud) para cada hemisferio. La figura 4.a y b muestra la evolución temporal mensual del DZD en función de las latitudes y del tiempo para las regiones norte y sur. La estacionalidad mostrada 
por la Climatología y las FEOs es claramente confirmada por los DZD costero. Además, al comparar ambas series se observa claramente que los desplazamientos están acoplados, aunque, muestren diferencias en amplitud. Por otro lado, estas series reportan claramente las diferencias de amplitud entre la región norte y sur, además, el desplazamiento del cinturón de los oestes en la región norte sigue la tendencia a desplazarse hacia altas latitudes a medida que avanza con el tiempo, aunque en la región sur, este comportamiento no están evidente.

\section{(a) Modo1 69,2\%}

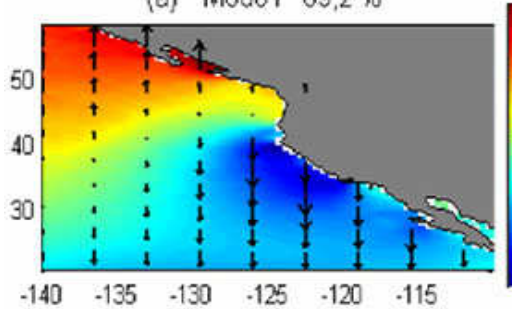

(c) Modo2 $15,83 \%$

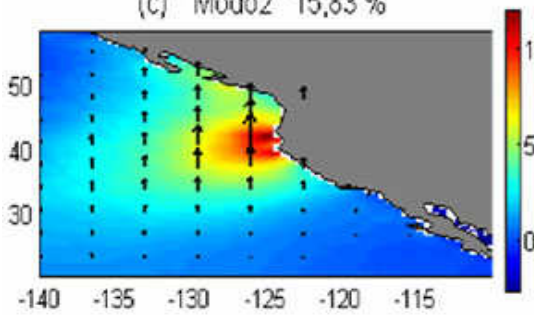

(b) Variabilidad Temporal del Esfuerzo del Viento Meridional del Modo1
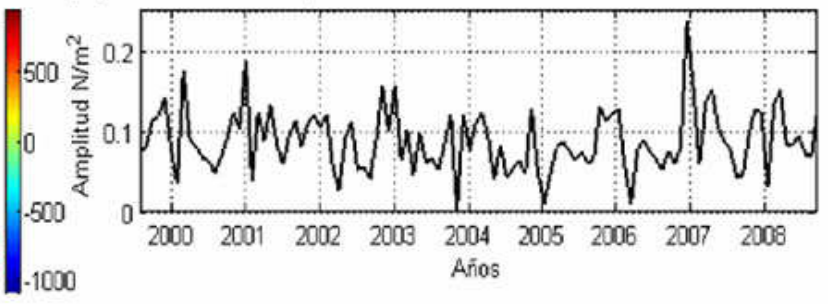

(d) Variabilidad Temporal del Esfuerzo del Viento Meridional del Modo2

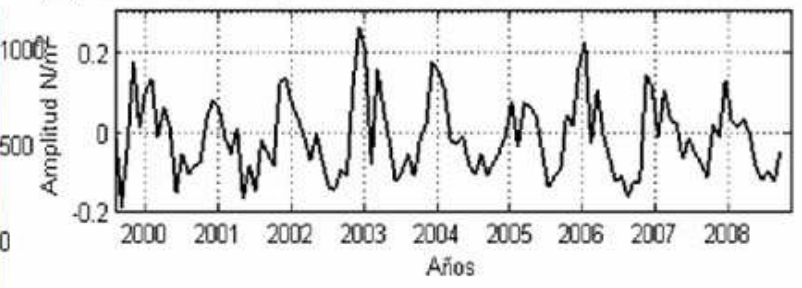

FIGURA 3. Funciones empíricas ortogonales para el esfuerzo del viento meridional, zona sur. Estructura espacial (a y c) y temporal (b y d) de los dos primeros modos.

FIGURE 3. Empirical orthogonal functions of meridional wind stress, southern area. Temporal (b and d) and spatial structure (a and $\mathrm{c}$ ) of the two first modes.

(a) Región Norte

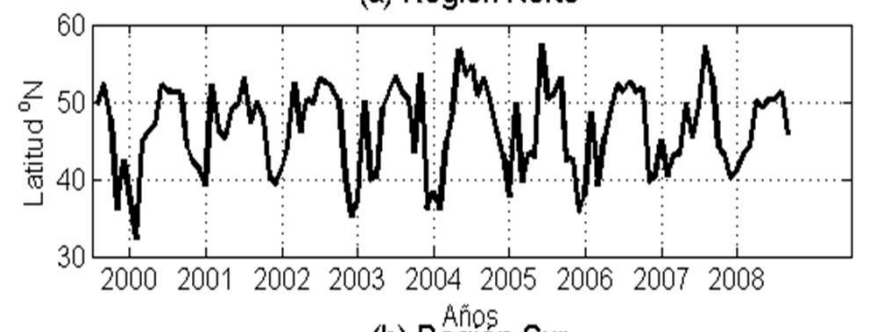

(b) Region Sur

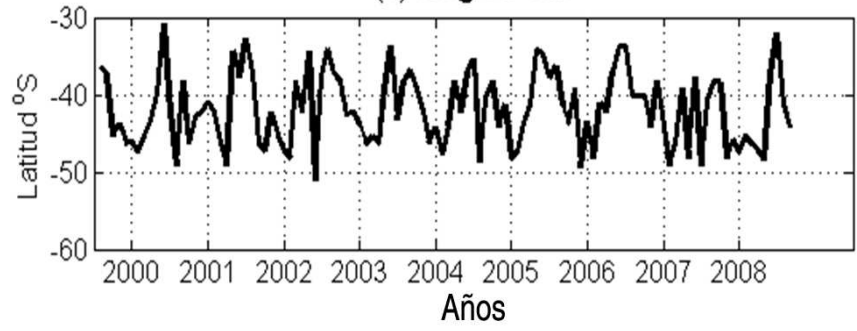

FIGURA 4. Evolución cronológica de las series de la zona de divergencia en latitudes (a) Región norte, (b) Región sur.

FIgURE 4. Temporal evolution of time series of the divergence zone in latitudes (a) Northern region, (b) Southern region. 
La aplicación de las Funciones de Autocorrelación es una buena herramienta para comprobar la estacionalidad de ciertas señales, como es el caso del DZD. En ambas serie las funciones verificaron la estacionalidad de ambas series. El cálculo posterior fue la aplicación de Correlaciones Cruzada, donde la figura 5 muestra claramente que ambas series están acopladas y al ser comparadas estacionalmente muestra un desfase de seis meses. Concluyendo, que ambas series están desfasadas en 6 meses o el desfase que existe entre las dos series para alcanzar el máximo desplazamiento hacia los polos o hacia el ecuador.

Posteriormente para comparar los DZD del esfuerzo del viento de ambos hemisferios y buscar la influencia de otras señales en el espacio del tiempo y las frecuencias, se estudiaron ambas series mediante la Transformada de Wavelet. Este método han sido desarrollado durante los últimos años (Terradellas et. al., 2001, 2003; Cuxart et al., 2002) y se destacan sobre los métodos tradicionales ya que la transformada de wavelet presenta una capacidad de filtrar distintas escalas muy superior al proceso de promediado, con lo cual desaparece la necesidad del mínimo espectral (Cuxart et al., 2002). Por otra parte el método puede ser puede ser aplicado a cualquier escala, con lo cual, los fenómenos del DZD puede ser estudiado de una forma totalmente distinta, pudiéndose analizar los efectos globales y los de sus componentes mas elementales (Terradellas et al., 2003)

La figura 6 muestra la potencia espectral de wavelet de la zona norte (a) y sur (b) en conjunto con las series temporales del DZD de ambas regiones, figuras 6.c y d, respectivamente. Se observa para las dos regiones (Fig. 6.a y b) la predominancia de la señal estacional del DZD donde la banda espectral es mayor (> 8 ), sin embargo, para la región norte el periodo estacional (1999-2008) es mas largo que la estacionalidad mostrada por la región sur (20012007). Además, en el espacio de las frecuencias y el tiempo (Fig 6.a y b), se destacan algunos núcleos en la frecuencia de 3 meses con una significativa potencia espectral $(\sim 3)$, para ambas regiones con periodicidad de dos años, aproximadamente. Para estudiar más en detalle las señales de bajas frecuencia, al DZD se le filtró la señal estacional mediante un filtro de paso bajo o promedio corrido, ya que en las figuras 6.a y .b estas señales no son perceptibles debido a que la potencia espectral estacional se lleva el mayor porcentaje de la energía y se encontró que el DZD efectivamente está influenciado por señales de baja frecuencia, es decir, el evento cálido de EL Niño 2002-2003 como se observaba en las mayores amplitudes (Figs. 2, 3 y 4).

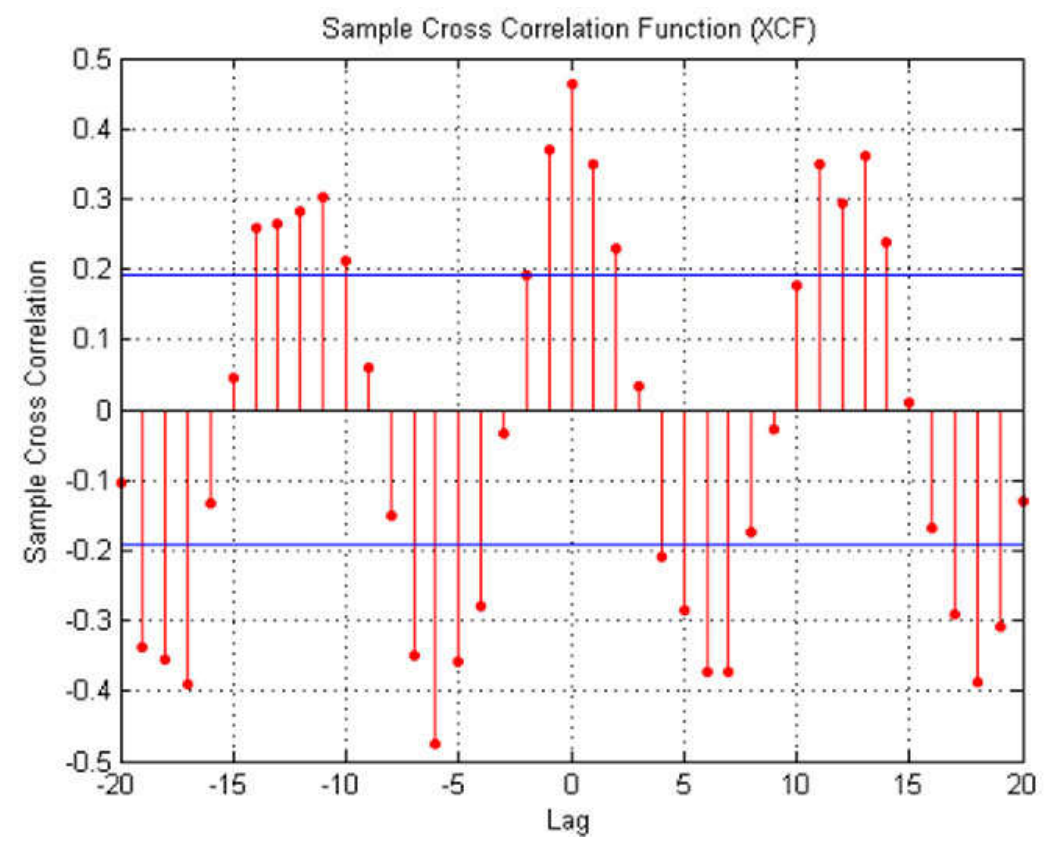

FIGURA 5. Correlación cruzada entre serie región norte y serie región sur.

FIGURE 5. Cross correlation between time series of northern and southern region. 

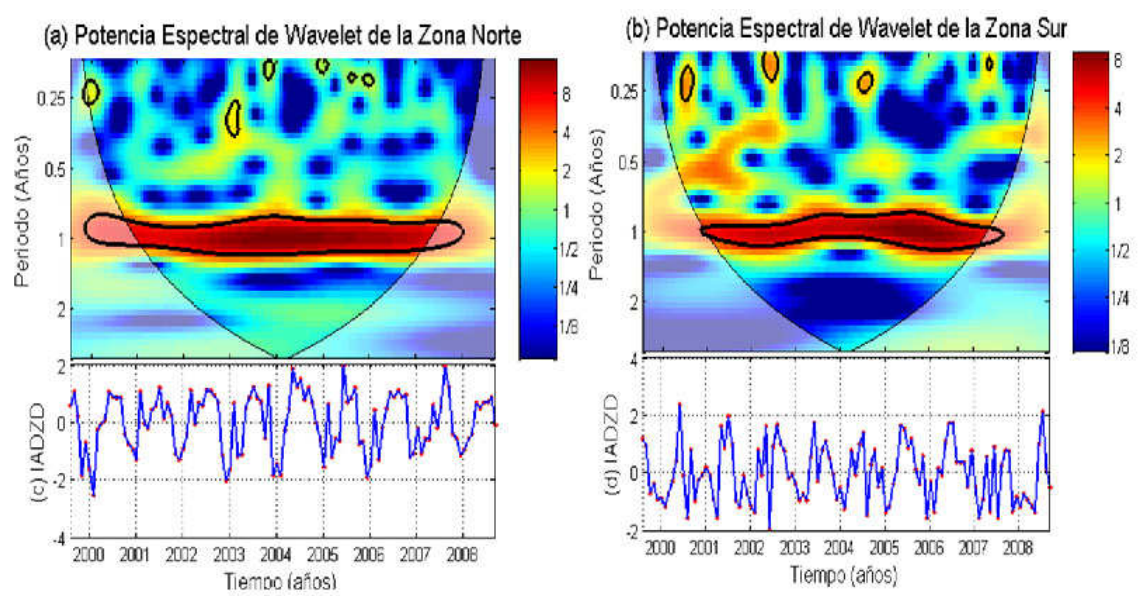

FIGURA 6. Potencia espectral de wavelet de la zona norte (a) y sur (b); Índice Promedio del Ancho del DZD [IADZD] para la zona norte $(\mathrm{c})$ y sur $(\mathrm{d})$.

FIGURE 6. Spectral potence of wavelet of the north zone (a) and south (b); wide means index of DZD [IADZD] for the north zone (c) and south (d).

\section{DISCUSIONES}

En este trabajo se analiza la serie del DZD mediante varios análisis estadísticos, los cuales rescatan dos señales de importancia, en las escalas instraestacional, estacional e interanual. Lo interesante de los resultados, es que a partir de dos series (norte y sur) de latitud versus tiempo, es posible predecir cómo se desplazan los centros de acción que invaden la gran cuenca del Océano Pacifico como las bajas presiones ecuatoriales, las altas presiones subtropicales y como los anticiclones Hawai-Pacífico Sur; las bajas presiones polares del frente polar; y las altas presiones polares (anticiclones ártico, antártico). Además, se observa en la figura 4 en la región norte un desplazamiento en verano con el tiempo hacia altas latitudes, de ser cierta esa tendencia estaríamos frente a los efecto del calentamiento global, es decir, donde los centros de altas presiones se están desplazándose hacia los polos, y lo cual explicaría el derretimiento acelerado de los polos.

A diferencia de la climatología la cual reportó que la zona de divergencia se desplaza $\sim 12^{\circ}$ latitud en el norte $\mathrm{y} \sim 10^{\circ}$ latitud en el sur, las FEOs revelaron en el segundo modo espacial del esfuerzo del viento meridional de la región sur que el desplazamiento latitudinal de la zona de divergencia es de $\sim 16^{\circ}$. Cabe mencionar que esta diferencia es explicable en término de que la climatología considera las dos componentes (zonal y meridional), en cambio el FEOs de esfuerzo del viento meridional está compuesta por solo una componente.

La cualidad de los FEOs es de sintetizar 110 imágenes mensuales en dos modos asociados a una estructura espacial y mostrar como evoluciona ésta mediante una estructura temporal, explicando a partir de estos dos modos la variabilidad espaciotemporal del DZD. Los resultados por los FEOs muestran que el DZD de ambas regiones tiene un comportamiento marcadamente estacional y con alguna influencia de otras escalas, en especial asociada a una señal interanual (El Niño 20022003), además de confirmar la estacionalidad del DZD mostrado en la climatología.

La Transformada Wavelet Continua en el espacio de las frecuencias mostró señales importantes tanto en las altas frecuencias, estacional e interanual. En las altas frecuencias se destacan pequeños núcleos observados en la región austral los cuales tienen cierta periodicidad ( $\sim 2$ años) atribuibles a la influencia del anticiclón antártico, dado que dicha zona está expuesto a una frontera abierta.

En la escala estacional el DZD muestra una señal espectral de alta energía, siendo la frecuencia más dominante de todas las señales, aunque, la zona norte muestra una mayor prolongación de la estacionalidad a través del tiempo que la región sur. Esta asimetría en tiempo de los DZD de ambos 
sistemas, nuevamente seria explicable en termino que la región sur es vulnerable a forzantes externos proveniente de altas latitudes.

\section{AGRADECIMIENTOS}

Al proyecto interno de la Dirección de Investigación de la Universidad del Bío-Bío No: 064307 3/R. Además, al Director del Dpto. de Física Francisco Brito por el apoyo brindado para hacer posible que los alumnos tesistas cuenten con un espacio acorde a las necesidades de una buena academia.

\section{BILIOGRAFIA}

Bakun, A. y C. S. Nelson. 1991. The seasonal cycle of wind stress curl in sub-tropical eastern boundary current regions. Journal of Physical Oceanography, 21, 1815-1834.

Barnett, T. P. \& W. C. Patzert. 1980. Scales of theral varibility in the tropical Pacific. Journal Geophysical Research. 10: 529-540.

Beardsley, R., C. Dorman, A. Frieche, K. Rosenfeld, y C. Winnant. 1987. Local Atmospheric during the Coastal Ocean Dynamical Experiment. A description of the marine boundary layer and atmospheric condictions over a northern California upwelling region. Journal of Geophysical Research, 92, 1647-1488

Carvajal, B., 1994. Transporte y turbulencia y su incidencia en el reclutamiento de la anchoveta (Engraulis ringers) en la Zona Norte de Chile $\left(20^{\circ} \mathrm{S}\right)$. Tesis Biólogo Pesquero, Universidad Arturo Prat. Iquique, Chile.

Cuxart, J., Morales, G., Terradellas, E., y Yague, C. 2002. Study of coherente structures and estimation of the pressure transport terms for nocturnal stable boundary layer, Boundary-Layer Meteorol. 102, 305-328.

FuenZalida, R. 1992. Proceso de surgencia en la región norte de Chile, latitudes $20^{\circ} 30^{\prime} \mathrm{S}-21^{\circ} 45^{\prime} \mathrm{S}$.
Investigaciones Científica y Tecnología. Serie Ciencias Mararinas, 2, 79-103.

Hickey, B. M., 1979. The California Current System, hypotheses and facts. Progress in Oceanography., 191-279.

KeLLY, K. A. 1985. The influence of winds and topography on the surface temperature patterns over the California slope. Journal Geophysical Research. 90: 11,783-11,798.

Montecinos, A., 1991. El efecto del fenómeno de El Niño en los vientos favorables a la surgencia costera. Oceanographer thesis, Universidad Católica de Valparaíso, Chile.

Pizarro, O., S. Hormazábal, A. González y E. Yañez, 1994. Variabilidad del viento, nivel del mar y temperatura en la costa norte de Chile. Invest. Mar., Valparaíso, 22: 85-101.

REID, J. L. 1965. Intermediate waters of the Pacific Ocean. The Johns Hopkins Oceanographic Studies, 2:85 pp.

RutLlANT, J. 2004.Aspecto de la circulación a gran escala asociada al ciclo ENOS 1997-1999 y sus consecuencias en régimen de precipitación en Chile. Edic El Niño-La Niña 1997-2000. Sus Efectos en Chile. CONA, Chile, Valparaíso, pp: 61:76.

SaAvedra, N. y A. J. Foppiano. 1992. Contribución a la cinemática del anticiclón del Pacífico Sur. Geoacta, 19:95-110.

Silva, N. y S. Neshyba. 1977. Corrientes superficiales frente a la costa austral de Chile. Cienc. Tecnol. Mar, 3:37-42.

SCHWERDTFEgER, W., 1976. Climates of Central and South America. In World Survey of Climatology, Vol. 12, W. Wchwerdtfeger, ed. Elsevier, Amsterdam.

Terradellas, E., Morales, G., Cuxart, J., y Yague, C. 2001. Wavelet Method: Application to study of th estable atmospheric Boundary layer under nonstationary conditions. Dyn. Atmos. Ocean 34, 225-244.

Terradellas, E., Soler, M. R., Ferreres, E., y Bravo, M. 2003. Analysis of oscillations in the atmosfheric stable Boundary-layer using wavelet methods. Boundary-Layer Meteorol. C.Torrence y Gilbert P. Compo "A Practical Guide to Wavelet Analysis", 1997.

WYRTKY, K. 1975. Fluctuation of the dynamic topography in the Pacific Ocean. J. Phys. Oceanogr., 450-459. 